\title{
The acute respiratory distress syndrome
}

\author{
Michael A. Matthay, ${ }^{1}$ Lorraine B. Ware, ${ }^{2}$ and Guy A. Zimmerman ${ }^{3}$ \\ ${ }^{1}$ Cardiovascular Research Institute and Departments of Medicine and Anesthesia, UCSF, San Francisco, California, USA \\ 2Division of Allergy, Pulmonary and Critical Care Medicine, Department of Medicine, Vanderbilt University, Nashville, Tennessee, USA. \\ ${ }^{3}$ Department of Medicine, Division of Pulmonary and Critical Care Medicine, University of Utah, Salt Lake City, Utah, USA.
}

\begin{abstract}
The acute respiratory distress syndrome (ARDS) is an important cause of acute respiratory failure that is often associated with multiple organ failure. Several clinical disorders can precipitate ARDS, including pneumonia, sepsis, aspiration of gastric contents, and major trauma. Physiologically, ARDS is characterized by increased permeability pulmonary edema, severe arterial hypoxemia, and impaired carbon dioxide excretion. Based on both experimental and clinical studies, progress has been made in understanding the mechanisms responsible for the pathogenesis and the resolution of lung injury, including the contribution of environmental and genetic factors. Improved survival has been achieved with the use of lung-protective ventilation. Future progress will depend on developing novel therapeutics that can facilitate and enhance lung repair.
\end{abstract}

\section{Introduction}

Since the original description of the acute respiratory distress syndrome (ARDS) in 1967, considerable progress has been made in understanding the pathogenesis and pathophysiology of acute lung injury (ALI) (1-4). The likelihood of survival is determined by the severity of lung injury, the extent of nonpulmonary organ dysfunction, preexisting medical conditions, and the quality of supportive care. Because ARDS is a complex syndrome with a broad clinical phenotype, it has been challenging to translate the results of cell and animal studies to pharmacologic therapies that reduce mortality in humans. Nevertheless, laboratory-based investigations have produced valuable insights into the mechanisms responsible for the pathogenesis and resolution of lung injury, and preclinical studies paved the way for important improvements in supportive care. Two of these therapies, lung-protective ventilation and fluid-conservative management, have reduced mortality and morbidity, respectively. This review of ARDS will focus on some of these issues, including new insights into the molecular mechanisms of lung injury and repair.

\section{Definitions, epidemiology, incidence, and mortality}

Criteria for the diagnosis of ARDS have evolved. The original description emphasized rapidly progressive respiratory failure from noncardiogenic pulmonary edema, requiring mechanical ventilation because of severe arterial hypoxemia and difficulty breathing (5). In 1988, a 4-point scoring system provided a quantitative assessment of lung injury severity based on the degree of hypoxemia, the level of positive end-expiratory pressure (PEEP), static respiratory compliance, and the extent of radiographic infiltrates (6), and this scoring system has been useful for research and clinical trials. In 1994, a consensus conference recommended simplified criteria: arterial hypoxemia with $\mathrm{PaO}_{2} / \mathrm{FiO}_{2}$ ratio less than $300 \mathrm{mmHg}$ and less than $200 \mathrm{mmHg}$ to define ALI and ARDS, respectively, and bilateral radiographic opacities without evidence of left atrial hypertension (7). These criteria have been widely utilized, although some investigators believe that the definitions should specify the level of PEEP and/or the fraction of inspired oxygen. A recent report - what is now called the Berlin definition - recommends use of three categories of ARDS, based

Conflict of interest: The authors have declared that no conflict of interest exists. Citation for this article: J Clin Invest. 2012;122(8):2731-2740. doi:10.1172/JCI60331. on the degree of hypoxemia: mild $\left(200 \mathrm{mmHg}<\mathrm{PaO}_{2} / \mathrm{FiO}_{2} \leq 300\right.$ $\mathrm{mmHg})$, moderate $\left(100 \mathrm{mmHg}<\mathrm{PaO}_{2} / \mathrm{FiO}_{2} \leq 200 \mathrm{mmHg}\right)$, and severe $\left(\mathrm{PaO}_{2} / \mathrm{FiO}_{2} \leq 100 \mathrm{mmHg}\right)(8)$. Whether stratification of patients based on these descriptions will advance the efficacy of clinical detection and of charting the natural history of ARDS remains to be determined.

Most investigations have focused on ALI and/or ARDS patients who are already mechanically ventilated. Recently, progress has been made in diagnosing ALI in spontaneously breathing patients who have bilateral pulmonary infiltrates and arterial hypoxemia and in whom intravascular volume overload and congestive heart failure are excluded $(9,10)$. This approach facilitates patient identification and testing of new therapies prior to the need for mechanical ventilation. Figure 1 provides a clinical vignette describing early recognition of ALI.

Bacterial or viral pneumonia is the most common cause of ALI and ARDS (1). Sepsis due to nonpulmonary infections, aspiration of gastric contents, and major trauma with shock also commonly precipitate the injury. Less commonly, acute pancreatitis, transfusions, drug reactions, and fungal and parasitic lung infections are linked to ALI and ARDS. The coexistence of two or more of these risk factors can enhance the likelihood of developing ALI or ARDS (1).

A prospective epidemiologic study in 1999-2000 estimated an annual incidence of ALI and ARDS of 190,000 adult patients in the United States (11). There is a substantial incidence of ALI and ARDS in children as well $(12,13)$. Data from 2001-2008 indicate that the incidence of ALI and/or ARDS in hospitalized adults has declined, perhaps secondary to more widespread use of lung-protective ventilation, reductions in nosocomial infections, and more conservative use of blood products $(14,15)$.

Severe arterial hypoxemia $\left(\mathrm{PaO}_{2} / \mathrm{FiO}_{2}<100\right)$ and an increase in the pulmonary dead space fraction $(>0.60)$ are associated with higher mortality (16), as are shock, liver dysfunction, acute kidney injury, age over 60 years, and higher severity of illness scores (17-19). Based on the NIH Heart, Lung and Blood Institute (NHLBI) ARDS Network trials, 60-day mortality has declined from $36 \%$ in $1996-1997$ to $26 \%$ in $2004-2005$ (20). The most recent ARDS Network clinical trials reported a 60-day mortality of $22 \%$ in adult patients despite higher APACHE III scores and a higher incidence of shock at enrollment compared with a prior trial in 2006 (Figure 2 and ref. 4). 

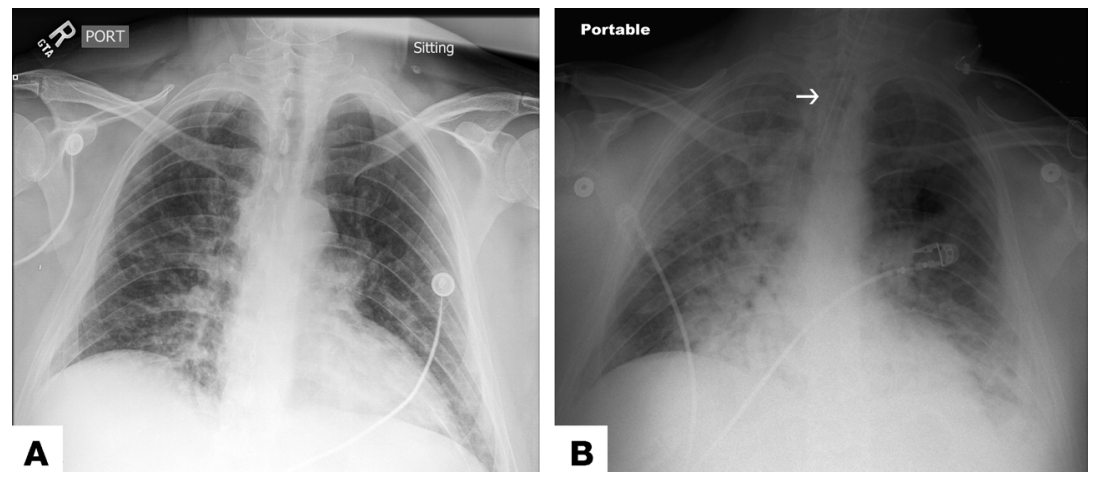

\section{Figure 1}

Chest radiograph of a patient with influenza-related pneumonia that illustrates early ALI, which progressed over 48 hours to more classic ALI that required positive-pressure ventilation. (A) Anterior-posterior portable chest radiograph of a previously healthy 41-year-old man who presented to the emergency department with a 2-day history of myalgias and fever, a productive cough, and shortness of breath. Chest auscultation revealed rales and rhonchi posteriorly in both lung fields. The chest radiograph demonstrates patchy infiltrates in the right lower lung field and also in the left lower lung field. (B) Anterior-posterior chest radiograph 48 hours after the chest radiograph in A, 1 hour after endotracheal intubation (arrow) and initiation of positive-pressure ventilation using the ARDS Network lung-protective ventilation protocol (97). There was marked progression of the bilateral radiographic infiltrates, with dense consolidation in the right upper, right lower, and left lower lung zones. The patient's hypoxemia steadily worsened during the 48 hours following his initial presentation, accompanied by an increase in respiratory rate to 40 breaths/minute. Diagnostic evaluation confirmed H1N1 influenza infection. All cultures for bacteria were negative. Recent clinical investigation indicates that it is possible in some patients to diagnose ALI in an early phase (9), as shown in A, well before the progression of acute respiratory failure to the need for mechanical ventilation, as illustrated in B. Earlier diagnosis of ALI could facilitate testing of therapeutic strategies that may have timedependent efficacy prior to the development of established ALI that requires intubation and mechanical ventilation.

\section{Environmental and genetic influences}

Environmental and genetic factors that contribute to susceptibility and severity of ALI and ARDS have emerged as a major research focus. Chronic alcohol abuse increases the risk of ALI and ARDS (21) and multiple organ failure in septic shock (22). Both active and passive cigarette smoke exposure, as quantified by plasma levels of cotinine, have been independently associated with the development of ALI after severe blunt trauma (23). The mechanisms may include priming effects of cigarette smoke exposure on the lung endothelium, alveolar epithelium, or inflammatory cells.

Variants in more than 25 genes have been associated with developing ALI and/or ARDS and with clinical outcomes (24) including common variants of genes that regulate inflammation, coagulation, endothelial cell function, reactive oxygen radical generation, and apoptosis (25-29) - all processes that are important in lung injury and repair $(2,30,31)$. For example, the Fas pathway modulates apoptosis, inflammation, and epithelial cell injury; in a candidate gene study, common genetic variants in Fas were associated with susceptibility to developing clinical lung injury (27). African Americans with ALI have a higher risk of death compared with white patients. A candidate gene study identified a functional T-46C polymorphism ( $\mathrm{rs} 2814778$ ) in the promoter region of the Duffy antigen/receptor for chemokines $(D A R C)$ gene that was associated with a $17 \%$ increase in 60 -day mortality in AfricanAmerican patients enrolled in ARDS Network clinical trials. Plasma interleukin-8 levels were increased in those individuals with the DARC polymorphism, supporting one mechanism contributing to a worse clinical outcome (29).

Genetic polymorphisms that predispose individuals to the injurious effects of specific bacteria or viruses may influence the development of ALI and ARDS. Indeed, several polymorphisms are associated with more severe pneumococcal, Legionella, and viral lung infections (32). The genetic factors that regulate the virulence of infecting pathogens also require more research (33) to relate the severity of clinical lung injury to specific microbiologic variables that contribute to severe pneumonia and ALI. Genetic features of both the host and the microbe likely are important in determining the severity of lung injury.

In spite of the intriguing associations, putative causal genes require more validation in independent study populations, along with studies of gene and environmental interactions that may alter these associations. Genome-wide association studies for susceptibility and outcomes in multiple populations are in progress. One recent study of this nature identified PPFIA1 (which encodes liprin $\alpha$, a protein involved in cell adhesion, integrin expression, and cellmatrix interactions) as a predictor for developing acute lung injury after major trauma (25).

\section{Pathogenesis: dysregulated inflammation and alveolar barrier disruption}

Dysregulated inflammation, inappropriate accumulation and activity of leukocytes and platelets, uncontrolled activation of coagulation pathways, and altered permeability of alveolar endothelial and epithelial barriers remain central pathophysiologic concepts in ALI and ARDS (1-3). Activation of the innate immune response by binding of microbial products or cell injury-associated endogenous molecules (danger-associated molecular patterns [DAMPs]) to pattern recognition receptors such as the Toll-like receptors on the lung epithelium and alveolar macrophages is now recognized as a potent driving force for acute lung inflammation (34). Newly reported innate immune effector mechanisms, such as formation of neutrophil extracellular traps - lattices of chromatin and antimicrobial factors that capture pathogens but also can cause endothelial injury - and histone release by neutrophils (35) may contribute to alveolar injury. Signaling between inflammatory and hemostatic effector cells, such 


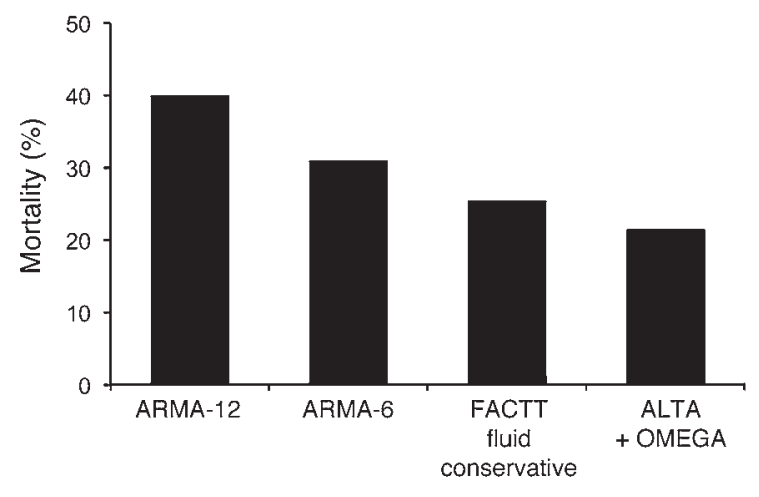

as platelet-neutrophil interaction, is important in some models, including acid-induced ALI, sepsis, and transfusion injury $(36,37)$. The delicate balance between protective and injurious innate and adaptive immune responses and hemostatic pathways may determine whether alveolar injury continues or is repaired and resolved. For example, in lung infection, acute inflammatory responses to pathogens and their toxins (38-40) cause ALI through leukocyte protease release, generation of reactive oxygen species, rampant synthesis of chemokines and cytokines, Toll-like receptor engagement, and actions of lipid mediators (33, 41, 42). Nevertheless, these same inflammatory mechanisms, when controlled rather

\section{Figure 2}

Mortality in ALI and ARDS. Shown is the 60-day mortality reported over the last 11 years in randomized clinical trials from the ARDS Network. ARMA-12 refers to the mortality rate of 431 patients enrolled into the higher-tidal volume arm (12 $\mathrm{ml}$ tidal volume/kg predicted body weight), and ARMA- 6 refers to the mortality of 430 patients enrolled in the lower-tidal volume arm ( $6 \mathrm{ml}$ tidal volume/kg predicted body weight) of one study (97). FACTT fluid conservative refers to the mortality of the 500 patients enrolled into the fluid-conservative arm of the Fluid and Catheter Treatment Trial (120). ALTA and OMEGA refer to the combined mortalities of the 2 most recent trials ( $N=517$ in both trials combined), Albuterol for the Treatment of ALI (136) and Omega-3 Fatty Acid, Gamma-Linolenic Acid, and Antioxidant Supplementation in the Management of ALI or ARDS (138).

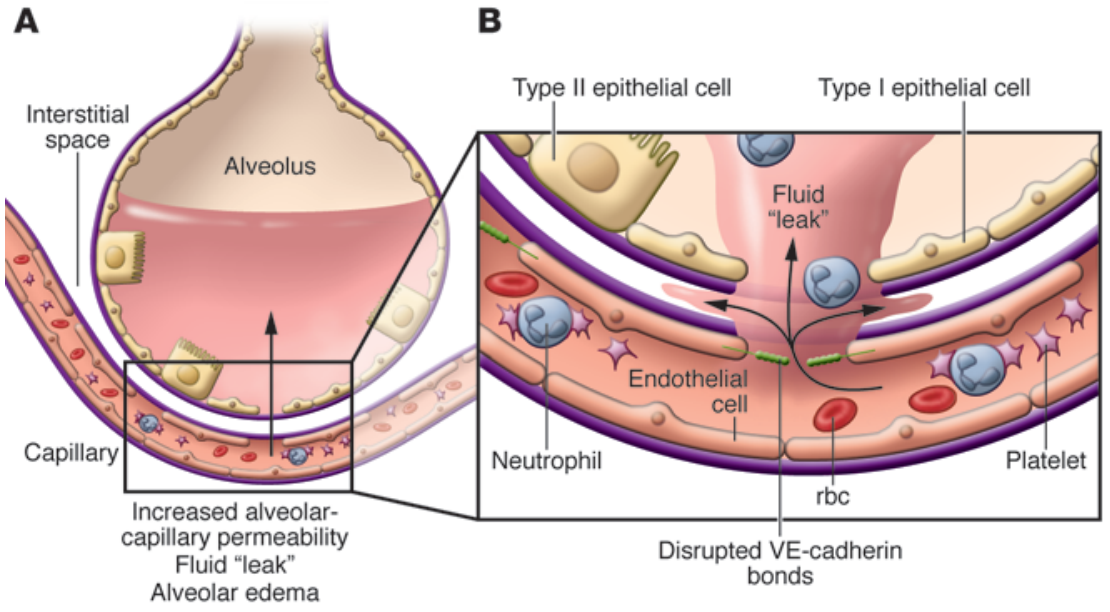

Figure 3

Molecular targets for new therapies that can lead to endothelial and epithelial barrier stabilization and reversal of increased permeability. (A) Disrupted alveolar barrier function, resulting in increased permeability to water, proteins, and other solutes, is a hallmark of clinical and experimental ALI. Intra-alveolar accumulation of neutrophils, other leukocytes, and erythrocytes is also associated with altered endothelial and epithelial barrier function. TNF- $\alpha$, IL-1, thrombin, and microbes and their toxins - including LPS, noxious agents, and factors generated by neutrophils and plateletleukocyte interactions - can destabilize and disrupt alveolar barrier function, leading to increased permeability. (B) Disruption of VE-cadherin bonds is a central mechanism of altered endothelial barrier function in experimental ALI and in models of sepsis and systemic vascular destabilization. VE-cadherin is an endothelial-specific adherens junction protein that mediates $\mathrm{Ca}^{2+}$-dependent homophilic interactions at the lateral cell membranes of adjacent endothelial cells. VE-cadherin is regulated by cytoplasmic associations with catenins and actin and by cytoskeletal organization, in addition to intracellular signaling by Rho and Rac. Disruption of VE-cadherin bonds also facilitates transendothelial migration of leukocytes and, in some studies, is associated with accumulation of leukocytes and platelets in microvessels. (C) Stabilizing agonists (i) or small-molecule mimetics bind to stabilizing receptors (ii) on endothelial cells in alveolar and systemic vessels, restoring barrier integrity. Stabilizing agonists include S1P, Slit2N, Ang1, atrial natriuretic peptide, APC, and ATP; multiple intracellular pathways and mechanisms are implicated (iii) (reviewed in refs. 58,61, 64). These intracellular mechanisms favorably influence cytoskeletal architecture, preserve catenin-VE-cadherin cytoplasmic interactions, prevent VE-cadherin internalization, and/or promote adherens junction formation (iv and v). 

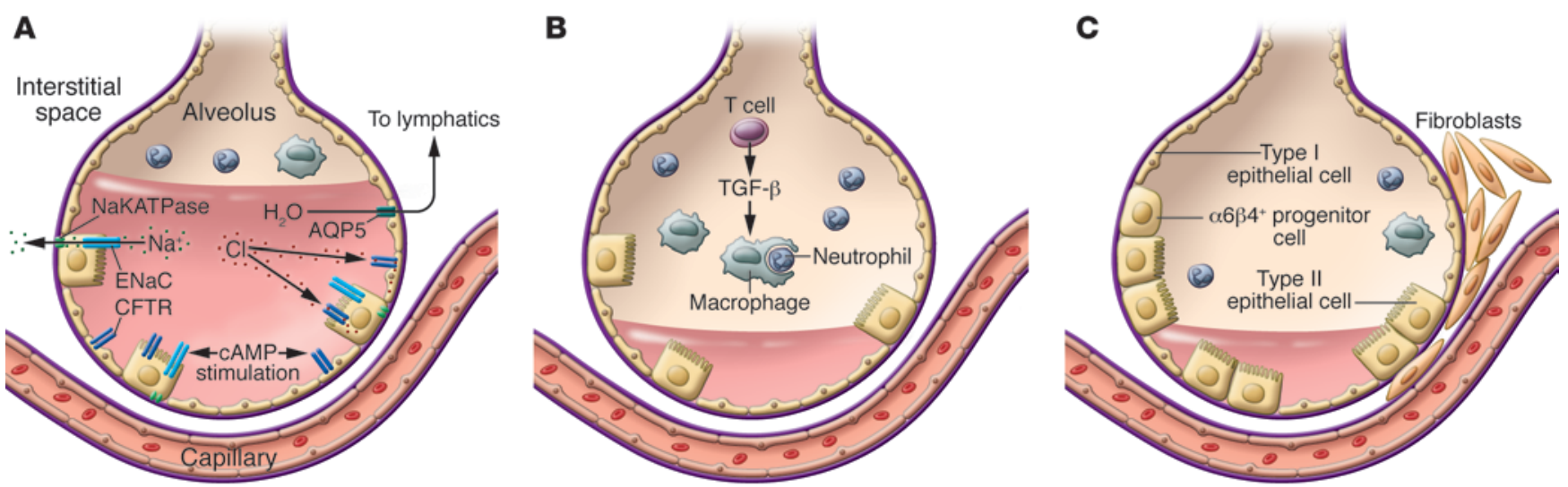

Figure 4

Resolution of ALI requires removal of alveolar edema fluid, removal of the acute inflammatory cells, and repair of the injured alveolar epithelium. (A) Alveolar edema fluid reabsorption is driven by vectorial transport of sodium and chloride from the airspaces to the lung interstitium, creating a mini-osmotic gradient. Sodium is transported across apical sodium channels (including epithelial sodium channel [ENaC]) and then extruded basolaterally by sodium-potassium ATPase (NaKATPase). Chloride is transported by transcellular or paracellular pathways. In the presence of endogenous or exogenous cAMP stimulation, the rate of alveolar fluid transport increases substantially, accomplished by increased expression and activity of ENaC, NaKATPase, and opening of the CFTR. For net fluid clearance to occur, however, there needs to be a reasonably intact alveolar epithelial barrier (see C). AQP5, aquaporin 5. (B) The resolution of inflammation in ALI and ARDS requires the removal of neutrophils from the distal airspace of the lung. Neutrophils are normally taken up by alveolar macrophages, a process termed efferocytosis. The rate of neutrophil clearance can be accelerated by regulatory T lymphocytes, in part by release of TGF- $\beta$. (C) Restoration of the alveolar epithelial barrier initially occurs by reepithelialization of the epithelial surface by alveolar type II cells. Although it was previously thought that this occurred via proliferation of resident type II cells, new work suggests there may be niches of progenitor cells that also contribute. An $\alpha 6 \beta 4^{+}$progenitor cell has been identified in the mouse lung that is responsible for restoration of the alveolar epithelial barrier after bleomycin-induced lung injury (88). Thus, repair may occur by endogenous stem cell proliferation, not just by epithelial cell migration and proliferation of existing differentiated cells.

46). Increased permeability is also linked to transfer of leukocytes and erythrocytes into the alveolar space in ARDS (46), as well as to inflammasome-regulated cytokines (47). A variety of mediators, pathways, and molecular systems contribute to altered alveolar endothelial and epithelial permeability (48-53). Vascular endothelial cadherin (VE-cadherin), an adherens junction protein, is critical for maintenance of endothelial barrier integrity in lung microvessels (54). Disruption of VE-cadherin homophilic bonds destabilizes lung microvascular barrier function (Figure 3B). Antibodies against VE-cadherin, destabilizing agonists such as TNF, thrombin, and VEGF, and leukocyte signals all interrupt VE-cadherin bonds and induce lung edema formation $(54,55)$. In contrast, stabilization of VE-cadherin bonds (Figure 3C) through genetic manipulation of VE-cadherin-catenin interactions (56) or prevention of dissociation of a phosphatase from VE-cadherin (57) reduces BAL protein and leukocytes in LPS-challenged mice. Thus, experimental manipulation of VE-cadherin alters alveolar and systemic endothelial barrier function and leukocyte transmigration, with pathogenic implications for clinical ALI and ARDS.

Molecular approaches to specifically reverse increased-permeability pulmonary edema have been long sought in ALI and ARDS research $(1,3)$. Candidate pathways for stabilization of lung and systemic endothelial barriers (reviewed in ref. 58) have recently been described. Systemic endothelium may be a critical therapeutic target in septic ALI (58) and in multiple organ failure associated with ALI and ARDS (1). A central therapeutic paradigm involves administration of stabilizing ligands that bind to receptors on endothelial cells and activate intracellular pathways, mediating cytoskeletal reorganization and catenin-VE-cadherin interactions that tighten VE-cadherin bonds (Figure 3C). Sphingosine-1-phosphate $(\mathrm{S} 1 \mathrm{P})$ is a lipid recognized by $\mathrm{G}$ protein-coupled receptors on endothelial cells (S1Pr1, S1Pr2, S1Pr3). S1P binding to S1Pr1 induces actin cytoskeletal reorganization, RAC activation, localization of $\alpha$-, $\beta$-, and $\gamma$-catenin and VE-cadherin to regions of intercellular contact, and assembly of adherens junctions in cultured human endothelial cells $(59,60)$. S1P enhances pulmonary and systemic endothelial barrier integrity in vivo and in vitro (reviewed in ref. 61), and small-molecule agonists of endothelial S1Pr $\mathrm{Pu}_{1}$ supress cytokine storm and lung leukocyte recruitment in experimental influenza (62). S1P is present in high concentrations in plasma and in that compartment regulates basal and inflammation-triggered vascular leak in the lungs and systemic vessels of mice (63). Platelets may locally contribute S1P at sites of vascular injury (60) and may reduce alveolar hemorrhage - another complication of endothelial barrier disruptions (46) - under some conditions; this effect may in part be related to delivery of S1P. However, effects of S1P or synthetic S1P receptor agonists may depend on biologic context (60) and time/duration of administration (64), since $\mathrm{S} 1 \mathrm{Pr}_{2}$ and $\mathrm{S} 1 \mathrm{Pr}_{3}$ are barrier destabilizing and $\mathrm{S} 1 \mathrm{Pr}_{1}$ undergoes time-dependent desensitization $(60,63)$. Similarly, other receptor-mediated signaling systems, such as those recognized by thrombin and other $\mathrm{G}$ protein-coupled protease-activated receptor agonists, may differentially trigger lung endothelial barrier disruption or stabilization, depending on time and context (65).

The Robo4/Slit signaling system also stabilizes the endothelial barrier $(66,67)$. In contrast to S1P receptors (60), Robo4 expression is restricted to endothelial cells (68). An active fragment of the Robo4 ligand Slit (Slit2N) inhibits tyrosine phosphorylation of VEcadherin and preserves its association with P120 catenin, preventing VE-cadherin internalization and abnormal permeability of human microvascular endothelial cells induced by TNF- $\alpha$, IL-I, or LPS (67). In mice, Slit $2 \mathrm{~N}$ reduces pulmonary and systemic vascular perme- 
Table 1

Selected clinical trials of ALI and ARDS

\begin{tabular}{|c|c|c|c|c|}
\hline Intervention & Reference & Study phase & Study population ${ }^{A}$ & Results \\
\hline Lung-protective ventilation & 96 & Phase III & $\operatorname{ARDS}(N=53)$ & Decrease in mortality \\
\hline Lung-protective ventilation & 97 & Phase III & $\operatorname{ARDS}(N=861)$ & Decrease in mortality \\
\hline Lung-protective ventilation & 98 & Phase III & $\operatorname{ARDS}(N=103)$ & Decrease in mortality \\
\hline High PEEP & 108 & Phase III & $\operatorname{ARDS}(N=549)$ & No difference in mortality \\
\hline High PEEP & 109 & Phase III & $\operatorname{ARDS}(N=385)$ & No difference in mortality \\
\hline High PEEP & 110 & Phase III & $\operatorname{ARDS}(N=382)$ & No difference in mortality \\
\hline High-frequency ventilation & 116 & Phase II & $\operatorname{ARDS}(N=148)$ & No difference in mortality \\
\hline Prone position & 111 & Phase III & $\begin{array}{l}\text { ALI and ARDS in } \\
\text { children }(N=102)\end{array}$ & No difference in mortality \\
\hline Prone position & 112 & Phase III & $\operatorname{ARDS}(N=342)$ & No difference in mortality \\
\hline Neuromuscular blockade & 113 & Phase III & $\operatorname{ARDS}(N=340)$ & Decrease in mortality \\
\hline Esophageal pressure to adjust PEEP & 114 & Phase II & $\operatorname{ARDS}(N=61)$ & Improved oxygenation \\
\hline Surfactant & 125 & Phase III & $\operatorname{ARDS}(N=448)$ & No difference in mortality \\
\hline Methylprednisolone & 126 & Phase III & $\operatorname{ARDS}(N=99)$ & No difference in mortality \\
\hline Methylprednisolone & 127 & Phase III & $\operatorname{ARDS}(n=24)$ & Decrease in mortality, but small study \\
\hline Methylprednisolone & 128 & Phase III & $\operatorname{ARDS}(n=180)$ & No difference in mortality \\
\hline Methylprednisolone & 129 & Phase III & $\operatorname{ARDS}(N=91)$ & $\begin{array}{l}\text { Reduction in duration of mechanical } \\
\text { ventilation, but major limitations } \\
\text { related to study design }\end{array}$ \\
\hline Liposomal prostaglandin $\mathrm{E}_{1}$ & 130 & Phase III & $\operatorname{ARDS}(N=350)$ & No difference in mortality for results \\
\hline Antioxidants & 132 & Phase II & $\operatorname{ARDS}(N=46)$ & No difference in mortality \\
\hline Nitric oxide & 135 & Phase III & $\operatorname{ARDS}(N=385)$ & No difference in mortality \\
\hline$\beta_{2}$-Agonist (aerosolized) & 136 & Phase III & $\operatorname{ARDS}(N=282)$ & No difference in mortality \\
\hline$\beta_{2}$-Agonist (intravenous) & 137 & Phase III & $\operatorname{ARDS}(N=330)$ & No difference in mortality \\
\hline$\omega-3$ Fatty acid supplement & 138 & Phase III & $\operatorname{ARDS}(N=272)$ & No difference in mortality \\
\hline Pulmonary artery versus central venous catheter & 121 & Phase III & $\operatorname{ARDS}(N=1,000)$ & No difference in mortality \\
\hline Fluid-conservative versus fluid-liberal therapy & 120 & Phase III & $\operatorname{ARDS}(N=1,000)$ & $\begin{array}{l}\text { More ventilator-free days with } \\
\text { fluid-conservative therapy }\end{array}$ \\
\hline Extracorporeal membrane oxygenation & 115 & Phase III & $\operatorname{ARDS}(N=90)$ & $\begin{array}{l}\text { Decrease in mortality, but } \\
\text { results not conclusive }\end{array}$ \\
\hline APC & 134 & Phase III & Nonseptic ARDS $(N=75)$ & No difference in mortality \\
\hline APC & 133 & Phase III & Sepsis $(N=1,697)$ & No difference in mortality \\
\hline GM-CSF & 131 & Phase II & $\operatorname{ARDS}(N=130)$ & No difference in mortality \\
\hline
\end{tabular}

APhysiologic criteria for ALI and ARDS varied among these trials.

ability in LPS lung injury, cecal ligation and puncture, and influenza infection, increasing survival (67). Cytokine levels are not decreased, indicating that Robo4 signaling does not inhibit this component of inflammation and that endothelial barrier stabilization may be sufficient to improve outcomes in lethal infectious challenges $(58,67)$.

Although numerous endothelial-stabilizing agonists and intracellular pathways have been identified (refs. 58, 61, and Figure $3 \mathrm{C}$ ), use of these agents may have unintended consequences. Molecular mechanisms by which plasminogen activator inhibitor-1 mediates Pseudomonas-associated alveolar endothelial barrier disruption have been identified, but $\mathrm{Pail}^{-/-}$mice have a defect in alveolar neutrophil recruitment and increased mortality compared with wild-type animals (69). This emphasizes the daedal relationships between barrier integrity and leukocyte transmigration and the precarious tension between injurious and protective inflammatory mechanisms that may operate in ALI and ARDS.

In contrast to endothelium, less is known about the potential mechanisms of alveolar epithelial stabilization, although epithelial permeability is critical in alveolar flooding $(70,71)$ and leukocyte accumulation (52) and potentially critical for intra-alveolar fibrin deposition and hyaline membrane forma- tion (72). Epithelial barriers involve cadherin-mediated adherens junction bonds and tight junctions, although the topography differs from endothelium, and E-cadherin substitutes for VE-cadherin (54). Alveolar epithelial barriers are tighter than alveolar endothelial barriers (70), but the two have functional interactions (2). For example, under some (55), but not all (57), conditions, disruption of endothelial VE-cadherin bonds causes alveolar epithelial leak and epithelial injury. Mesenchymal stem cells (MSCs) (see Future directions) restore barrier integrity in cytokine-treated cultured human alveolar epithelial cells (73). The mechanism involves release of angiopoietin-1, which inhibits actin stress fiber formation and redistribution of the tight junction protein claudin 18 in epithelial cells (73) and also induces S1P production and inhibits endothelial VE-cadherin internalization (58).

\section{Resolution}

Resolution of ALI requires effective and synchronous (a) reabsorption of alveolar edema, (b) repair of the epithelial and endothelial barriers, and (c) removal of inflammatory cells and exudate from the distal airspaces (Figure 4). Resolution of ALI 


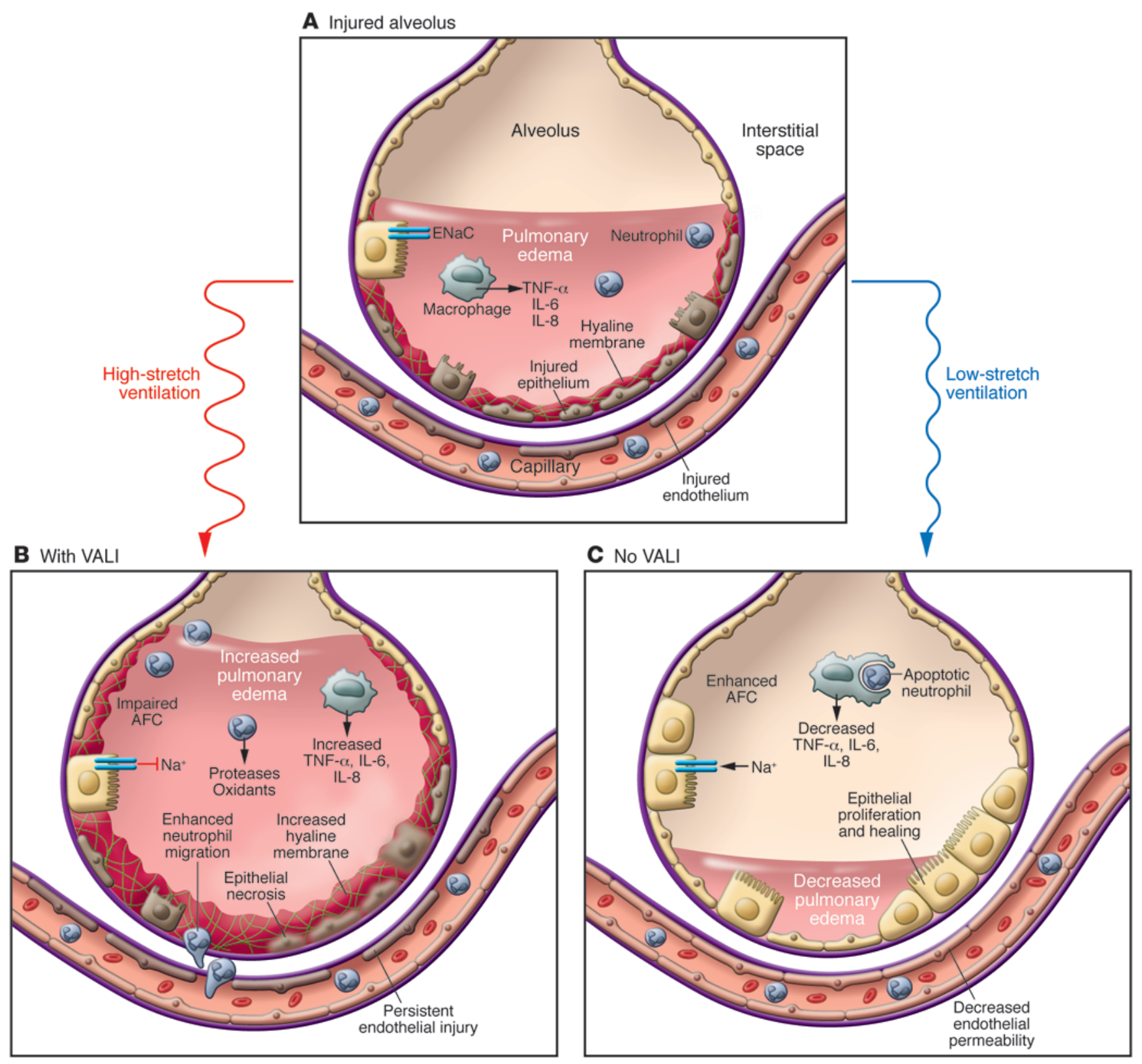

Figure 5

Mechanisms of ventilator-associated lung injury (VALI). (A) ALI leads to lung endothelial and epithelial injury, increased permeability of the alveolar-capillary barrier, flooding of the airspace with protein-rich pulmonary edema fluid, activation of alveolar macrophages with release of proinflammatory chemokines and cytokines, enhanced neutrophil migration and activation, and fibrin deposition (hyaline membranes). (B) If the injured lung is ventilated with high tidal volumes and high inflation pressures (high-stretch ventilation), then lung injury is exacerbated, with increased lung endothelial and epithelial injury and/or necrosis, enhanced neutrophil margination, release of injurious neutrophil products such as proteases and oxidants, increased release of proinflammatory cytokines from alveolar macrophages and the lung epithelium, increased fibrin deposition, and increased hyaline membrane formation. Injurious mechanical ventilation can also impair alveolar fluid clearance (AFC) mechanisms. (C) In contrast, a protective ventilatory strategy (low-stretch ventilation) can limit further lung endothelial and epithelial injury, reduce the release of proinflammatory cytokines, and enhance alveolar fluid clearance through the active transport of sodium and chloride across the alveolar epithelium (see Figure 4), thereby reducing the quantity of pulmonary edema and allowing endothelial and epithelial repair to occur. Epithelial repair occurs through migration, proliferation, and differentiation of alveolar epithelial type II cells to repopulate the denuded basement membrane. Acute inflammation resolves through apoptosis of neutrophils, which are phagocytosed by alveolar macrophages (see Figure 4). 
and repair of alveolar structures may - like the initiation of alveolar damage - depend on a precise balance of inflammatory interactions and molecular signaling. For example, hyaluronan fragments found in the serum of ALI patients trigger release of chemokines by macrophages but also interact with Toll-like receptors to deliver signals that limit epithelial apoptosis and promote reestablishment of epithelial integrity in experimental lung injury (74).

Reabsorption of alveolar edema (Figure 4A) occurs through vectorial transport of sodium and chloride across alveolar epithelial type I and II cells to create a mini-osmotic gradient to reabsorb water (75-77). This process is impaired in ALI and ARDS because of apoptosis and necrosis of alveolar epithelium $(31,46)$ and defects in transcellular ion transport induced by proinflammatory cytokines, oxidants, and hypoxia $(75,78-$ 81). Effective reabsorption of edema fluid from the air spaces requires reestablishment of an effective epithelial barrier (70, 82). Reepithelialization occurs initially by proliferation of type II cells (46), and the traditional view is that type II cells are the main source of new alveolar epithelial cells (83). Nevertheless, there is new evidence that progenitor cells may exist in strategic niches in bronchoalveolar junctions and that the alveolar epithelium can be activated to regenerate the epithelial and endothelial barriers (84-87). For example, $\alpha 6 \beta 4$-expressing progenitor cells have been identified that account for a substantial fraction of the type II cells that reepithelialize the injured alveolar barrier (Figure 4C and ref. 88). There may also be a human c-kit ${ }^{+}$lung stem cell capable of renewing all lung cells (89), although this hypothesis is controversial and requires validation. There has been progress in understanding how inflammation is resolved through clearance of alveolar neutrophils, monocytes, and necrotic debris including the contributions of lipid mediators such as lipoxin A4, resolvin E1, and other antiinflammatory pathways (Figure $4 \mathrm{~B}$ and refs. 45, 90). Macrophages remove apoptotic neutrophils and monocytes via molecular mechanisms that have recently been more clearly identified (91-93). A deficiency of alveolar macrophages worsens influenza-related pneumonia and lung injury in mice, leading to an increase in neutrophils and neutrophil extracellular traps (94). Lymphocytes also contribute to resolution. In a mouse model of endotoxin-induced lung injury, $\mathrm{CD} 4^{+} \mathrm{CD} 25^{+}$regulatory $\mathrm{T}$ cells that were recruited to the air spaces played an essential role in resolving inflammation by enhancing neutrophil apoptosis and suppressing cytokine secretion, in part by release of TGF- $\beta$ (95).

\section{New insights based on clinical trials}

Many clinical trials have assessed pharmacologic interventions, innovative strategies for positive-pressure ventilation, and other supportive approaches to ALI and ARDS treatment, advancing our understanding of the mechanism of the disease (Table 1).

Lung-protective ventilation. Three randomized clinical trials demonstrated that lung-protective ventilation with lower tidal volumes and airway pressures reduces mortality in ALI and ARDS (96-98). Why did this simple change in how we ventilate patients have such an impressive impact?

Lung-protective ventilation reduces accumulation of pulmonary edema by preserving barrier properties of the alveolar endothelium and alveolar epithelium (99-102). In rats, the rate of resolution of alveolar edema was 3-fold higher with 6 versus $12 \mathrm{ml} / \mathrm{kg}$ tidal volume, in part because of reduced epithelial cell injury (103). Reduc- tions in markers of lung epithelial injury have also been observed in clinical studies of reduced ventilation tidal volume (104). Lung-protective ventilation also downregulates mechanosensitive proinflammatory pathways, resulting in reduced neutrophil accumulation in the alveoli and lower plasma levels of IL-6, IL-8, and soluble TNF receptor 1 (refs. 105, 106, and Figure 5).

Lung-protective ventilation also benefits nonpulmonary organ function. For example, ventilation with high tidal volume in rabbits with acid-induced ALI causes apoptosis of renal tubular cells, an effect that is attenuated with lower tidal volume (107), and lower tidal volume ventilation was associated with an increase in renal failure-free days in patients with ARDS (97).

The effects of prone positioning, optimal levels of PEEP, highfrequency ventilation, neuromuscular blockade, measurement of esophageal pressure, and extracorporeal circuits to enhance carbon dioxide excretion have also been tested in clinical trials (refs. 108-116 and Table 1). These approaches have value as rescue therapies but have not achieved sufficient efficacy to recommend them as primary treatments (117).

Fluid-conservative therapy. Approximately 30 years ago, it was shown experimentally that reducing lung vascular hydrostatic pressures decreases lung edema in the setting of increased lung vascular permeability $(118,119)$. The clinical importance of this observation was confirmed when the ARDS Network reported in a 1,000-patient randomized clinical trial that a fluid-conservative strategy significantly reduced the average duration of mechanical ventilation by 2.5 days (120), a difference that was not affected by use of the pulmonary artery catheter (121). The primary beneficial mechanism can be explained by a favorable effect on Starling forces: lower vascular pressure reduces transvascular fluid filtration, particularly in the presence of increased lung vascular permeability. Also, animal studies indicate that reduced lung vascular pressure can attenuate lung endothelial translocation of P-selectin and accumulation of intravascular neutrophils (122). Plasma levels of angiopoietin-2 were lower in patients who were treated with a fluid-conservative versus a fluid-liberal strategy, which supports the hypothesis that an antiinflammatory mechanism explains this effect (123).

Other trials. Abnormalities of surfactant in ALI and ARDS include decreased production, alterations in phospholipid composition, and inhibition of surfactant function by exuded plasma proteins, oxygen radicals, and proteases (124). All these abnormalities promote atelectasis. Nevertheless, unlike the success of surfactantreplacement therapy for infant respiratory distress syndrome, clinical trials of surfactant replacement have not improved clinical outcomes in ALI and ARDS (ref. 125 and Table 1).

Although some animal studies support the potential efficacy of antiinflammatory therapies for decreasing lung injury, clinical trials have not demonstrated a convincing reduction in mortality using GM-CSF or glucocorticoids, antioxidants, or anticytokine therapies that were tested in patients with sepsis (126-132). Explanations for these outcomes are likely multifactorial, and these therapeutic strategies may merit reexamination as our understanding of subpopulations of patients with ALI and ARDS grows.

There has been considerable interest in the possibility that anticoagulant therapy may be effective in ALI and ARDS because of the close link between procoagulant and proinflammatory pathways $(133,134)$. Nevertheless, activated protein C (APC) did not reduce mortality in a small trial in nonseptic ALI and ARDS (134). Furthermore, a recent trial of APC has provided evidence that this anticoagulant and antiinflammatory agent does not have efficacy 
for patients with severe sepsis (133), the most lethal cause of ALI and ARDS, in spite of previous clinical and experimental support for its use (3). Strategies and rationale for anticoagulants for ALI and ARDS will now need to be reevaluated.

Although pulmonary hypertension and lung vascular injury are important features of ALI and ARDS, vasodilator therapies including prostaglandin E1 and nitric oxide have not reduced mortality (refs. 130, 135, and Table 1). Treatment to accelerate the resolution of pulmonary edema with aerosolized or intravenous $\beta$-adrenergic agonists also failed to improve survival $(136,137)$. Nutritional supplement with $\omega-3$ fatty acid may be harmful (138).

\section{Future directions}

Cell-based therapy with allogeneic human MSCs has emerged as a promising approach to therapy for ALI and ARDS (139-144). MSCs secrete multiple effector molecules, including antiinflammatory cytokines, growth factors, and antimicrobial peptides. These can reverse the major abnormalities of lung injury, including altered lung endothelial and epithelial permeability, impaired alveolar edema fluid clearance, dysregulated inflammation, and infection. In addition, in an experimental model, MSCs attached to the alveolar wall by connexin-43-based gap junctional channels and transferred mitochondria to endotoxin-injured alveolar epithelium, restoring alveolar ATP production and normalizing surfactant production and epithelial barrier properties (145). Also, the discovery that shortterm function can be achieved in repopulated decellularized lungs raises the prospect that basic understanding of lung regeneration (146), including signals from the lung matrix, may lead to novel strategies to enhance repair in the severely injured lung.

With declining mortality, clinical trials of new therapies will need to enroll patients with the highest predicted mortality (4) and include approaches that combine clinical and biological predictors $(147,148)$ to select the patients at highest risk. Clinical trials are in progress to test whether statin therapy can favorably modulate inflammation and restore barrier integrity in ALI and ARDS (Figure 3C). In animals, aspirin was effective in preventing acidinduced and transfusion-associated lung injury (37). Consequently, a clinical trial is planned to test the efficacy of aspirin in patients at high risk for developing ALI. There is also progress on developing more effective extracorporeal systems to support gas exchange for patients with very severe lung injury (149). Further research into the complex factors that influence the functional status of patients after recovery from ALI or ARDS (150) may also stimulate new approaches to attenuate long-term muscle and neurologic impairments that cause disabilities in ALI and ARDS survivors.

\section{Acknowledgments}

The authors thank Andrew Manies for his invaluable assistance in preparing the manuscript and Diana Lim for her excellent work with the illustrations. Also, grants provided support for M.A. Matthay (R01HL51854, R37HL51856, U01HL10871301, and R01HL101779 from the NHLBI; R01A1087674 and P01A1053194 from the National Institute of Allergy and Infectious Diseases), L.B. Ware (HL088263 and HL103836 from the NIH and an American Heart Association Established Investigator Award), and G.A. Zimmerman (5R37HL44525-23, 1RC1HL100121, and 5RO1HL09175403 from the NIH).

Address correspondence to: Michael A. Matthay, University of California, San Francisco, 505 Parnassus Ave, M-917, Box 0624, San Francisco, California 94143-0624, USA. Phone: 415.353.1206; Fax: 415.353.1990; E-mail: michael.matthay@ucsf.edu.
1. Ware LB, Matthay MA. The acute respiratory distress syndrome. NEngl J Med. 2000;342(18):1334-1349.

2. Matthay MA, et al. Future research directions in acute lung injury: summary of a National Heart, Lung, and Blood Institute working group. Am J Respir Crit Care Med. 2003;167(7):1027-1035.

3. Matthay MA, Zimmerman GA. Acute lung injury and the acute respiratory distress syndrome: four decades of inquiry into pathogenesis and rational management. Am J Respir Cell Mol Biol. 2005;33(4):319-327.

4. Spragg RG, et al. Beyond mortality: future clinical research in acute lung injury. Am J Respir Crit Care Med. 2010;181(10):1121-1127.

5. Ashbaugh DG, Bigelow DB, Petty TL, Levine BE. Acute respiratory distress in adults. Lancet. 1967;2(7511):319-323.

6. Murray JF, Matthay MA, Luce JM, Flick MR. An expanded definition of the adult respiratory distress syndrome. Am Rev Respir Dis. 1988;138(3):720-723.

7. Bernard GR, et al. The American-European Consensus Conference on ARDS. Definitions, mechanisms, relevant outcomes, and clinical trial coordination. Am J Respir Crit Care Med. 1994;149(3 pt 1):818-824.

8. The ARDS Definition Task Force. Acute respiratory distress syndrome: the Berlin definition. JAMA. 2012;307(23):2526-2533.

9. Levitt JE, Bedi H, Calfee CS, Gould MK, Matthay MA. Identification of early acute lung injury at initial evaluation in an acute care setting prior to the onset of respiratory failure. Chest. 2009;135(4):936-943.

10. Gajic O, et al. Early identification of patients at risk of acute lung injury: evaluation of lung injury prediction score in a multicenter cohort study. Am J Respir Crit Care Med. 2011;183(4):462-470.

11. Rubenfeld GD, et al. Incidence and outcomes of acute lung injury. NEngl J Med. 2005;353(16):1685-1693.

12. Flori HR, Glidden DV, Rutherford GW, Matthay
MA. Pediatric acute lung injury: prospective evaluation of risk factors associated with mortality. Am J Respir Crit Care Med. 2005;171(9):995-1001.

13. Randolph AG. Management of acute lung injury and acute respiratory distress syndrome in children. Crit Care Med. 2009;37(8):2448-2454.

14. Li G, et al. Eight-year trend of acute respiratory distress syndrome: a population-based study in Olmsted County, Minnesota. Am J Respir Crit Care Med. 2011;183(1):59-66.

15. Toy $P$, et al. Transfusion related acute lung injury: Incidence and risk factors. Blood. 2012;119(7):1757-1767.

16. Nuckton TJ, et al. Pulmonary dead-space fraction as a risk factor for death in the acute respiratory distress syndrome. $N$ Engl J Med. 2002; 346(17):1281-1286

17. Liu KD, et al. Predictive and pathogenetic value of plasma biomarkers for acute kidney injury in patients with acute lung injury. Crit Care Med. 2007;35(12):2755-2761.

18. Cooke CR, et al. Predictors of hospital mortality in a population-based cohort of patients with acute lung injury. Crit Care Med. 2008;36(5):1412-1420.

19. Brown LM, et al. A simple classification model for hospital mortality in patients with acute lung injury managed with lung protective ventilation. Crit Care Med. 2011;39(12):2645-2651.

20. Erickson SE, Martin GS, Davis JL, Matthay MA, Eisner MD, Network NNA. Recent trends in acute lung injury mortality: 1996-2005. Crit Care Med. 2009;37(5):1574-1579.

21. Moss M, Bucher B, Moore FA, Moore EE, Parsons PE. The role of chronic alcohol abuse in the development of acute respiratory distress syndrome in adults. JAMA. 1996;275(1):50-54.

22. Moss M, Burnham EL. Chronic alcohol abuse, acute respiratory distress syndrome, and multiple organ dysfunction. Crit Care Med. 2003; 31(4 suppl):S207-S212.

23. Calfee CS, et al. Active and passive cigarette smoking and acute lung injury after severe blunt trauma. Am J Respir Crit Care Med. 2011;183(12):1660-1665.

24. Gao L, Barnes KC. Recent advances in genetic predisposition to clinical acute lung injury. Am J Physiol Lung Cell Mol Physiol. 2009;296(5):L713-L725.

25. Christie JD, et al. Genome wide association identifies PPFIA1 as a candidate gene for acute lung injury risk following major trauma. PLOS ONE. 2012;7(1):e28268.

26. Meyer NJ, et al. ANGPT2 genetic variant is associated with trauma-associated acute lung injury and altered plasma angiopoietin-2 isoform ratio. $A m J$ Respir Crit Care Med. 2011;183(10):1344-1353.

27. Glavan BJ, et al. Genetic variation in the FAS gene and associations with acute lung injury. Am J Respir Crit Care Med. 2011;183(3):356-363.

28. Gong MN. Gene association studies in acute lung injury: replication and future direction. Am J Physiol Lung Cell Mol Physiol. 2009;296(5):L711-L712.

29. Kangelaris KN, et al. The association between a Darc gene polymorphism and clinical outcomes in African American patients with acute lung injury. Chest. 2012;141(5):1160-1169.

30. Herrero R, et al. The biological activity of FasL in human and mouse lungs is determined by the structure of its stalk region. J Clin Invest. 2011;121(3):1174-1190.

31. Albertine KH, et al. Fas and fas ligand are upregulated in pulmonary edema fluid and lung tissue of patients with acute lung injury and the acute respiratory distress syndrome. Am J Pathol. 2002;161(5):1783-1796.

32. Mizgerd JP. Acute lower respiratory tract infection. 
NEngl J Med. 2008;358(7):716-727.

33. Darwish I, Mubareka S, Liles WC. Immunomodulatory therapy for severe influenza. Expert Rev Anti Infect Ther. 2011;9(7):807-822.

34. Opitz B, van Laak V, Eitel J, Suttorp N. Innate immune recognition in infectious and noninfectious diseases of the lung. Am J Respir Crit Care Med. 2010;181(12):1294-1309.

35. Mantovani A, Cassatella MA, Costantini C, Jaillon S. Neutrophils in the activation and regulation of innate and adaptive immunity. Nat Rev Immunol. 2011;11(8):519-531.

36. Caudrillier A, et al. Platelets induce neutrophil extracellular traps in transfusion-related acute lung injury. J Clin Invest. 2012;122(7):2661-2671.

37. Looney MR, Nguyen JX, Hu Y, Van Ziffle JA, Lowell CA, Matthay MA. Platelet depletion and aspirin treatment protect mice in a two-event model of transfusion-related acute lung injury. J Clin Invest. 2009;119(11):3450-3461.

38. Diep BA, et al. Polymorphonuclear leukocytes mediate Staphylococcus aureus Panton-Valentine leukocidin-induced lung inflammation and injury. Proc Natl Acad Sci U S A. 2010;107(12):5587-5592.

39. Wiener-Kronish JP, Pittet JF. Therapies against virulence products of Staphylococcus aureus and Pseudomonas aeruginosa. Semin Respir Crit Care Med. 2011;32(2):228-235.

40. Lucas R, et al. Agonist of growth hormone-releasing hormone reduces pneumolysin-induced pulmonary permeability edema. Proc Natl Acad Sci U S A. 2012;109(6):2084-2089.

41. Rittirsch D, Flierl MA, Ward PA. Harmful molecular mechanisms in sepsis. Nat Rev Immunol. 2008;8(10):776-787.

42. Imai $Y$, et al. Identification of oxidative stress and Toll-like receptor 4 signaling as a key pathway of acute lung injury. Cell. 2008;133(2):235-249.

43. Imai Y, et al. Angiotensin-converting enzyme 2 protects from severe acute lung failure. Nature. 2005;436(7047):112-116.

44. Kuba K, et al. A crucial role of angiotensin converting enzyme 2 (ACE2) in SARS coronavirus-induced lung injury. Nat Med. 2005;11(8):875-879.

45. Serhan CN, et al. Resolution of inflammation: state of the art, definitions and terms. Faseb J. 2007;21(2):325-332.

46. Bachofen M, Weibel ER. Alterations of the gas exchange apparatus in adult respiratory insufficiency associated with septicemia. Am Rev Respir Dis. 1977;116(4):589-615.

47. Dolinay T, et al. Inflammasome-regulated cytokines are critical mediators of acute lung injury. Am J Respir Crit Care Med. 2012;185(11):1225-1234.

48. Pittet JF, et al. TGF-beta is a critical mediator of acute lung injury. J Clin Invest. 2001;107(12):1537-1544.

49. Xu J, et al. Extracellular histones are major mediators of death in sepsis. Nat Med. 2009;15(11):1318-1321.

50. Bhandari V, et al. Hyperoxia causes angiopoietin 2 -mediated acute lung injury and necrotic cell death. Nat Med. 2006;12(11):1286-1293.

51. Looney MR, Su X, Van Ziffle JA, Lowell CA, Matthay MA. Neutrophils and their Fc gamma receptors are essential in a mouse model of transfusion-related acute lung injury. J Clin Invest. 2006 116(6):1615-1623.

52. Zemans RL, Colgan SP, Downey GP. Transepithelial migration of neutrophils: mechanisms and implications for acute lung injury. Am J Respir Cell Mol Biol. 2009;40(5):519-535.

53. Calfee CS, Matthay MA. Clinical immunology: Culprits with evolutionary ties. Nature. 2010; 464(7285):41-42.

54. Vestweber D, Winderlich M, Cagna G, Nottebaum AF. Cell adhesion dynamics at endothelial junctions: VE-cadherin as a major player. Trends Cell Biol. 2009;19(1):8-15.

55. Corada M, et al. Vascular endothelial-cadherin is an important determinant of microvascular integrity in vivo. Proc Natl Acad Sci US A. 1999;96(17):9815-9820.

56. Schulte D, et al. Stabilizing the VE-cadherin-catenin complex blocks leukocyte extravasation and vascular permeability. EMBO J. 2011;30(20):4157-4170.

57. Broermann A, et al. Dissociation of VE-PTP from VE-cadherin is required for leukocyte extravasation and for VEGF-induced vascular permeability in vivo. J Exp Med. 2011;208(12):2393-2401.

58. Goldenberg NM, Steinberg BE, Slutsky AS, Lee WL. Broken barriers: a new take on sepsis pathogenesis. Sci Transl Med. 2011;3(88):88ps25.

59. Lee MJ, et al. Vascular endothelial cell adherens junction assembly and morphogenesis induced by sphingosine-1-phosphate. Cell. 1999;99(3):301-312.

60. Obinata H, Hla T. Sphingosine 1-phosphate in coagulation and inflammation. Semin Immunopathol. 2012;34(1):73-91.

61. Wolfson RK, Lang G, Jacobson J, Garcia JGN. Therapeutic strategies to limit lung endothelial cell permeability. In: Voelkel N, Rounds S. The Pulmonary Endothelium: Function in health and disease. Hoboken, New Jersey, USA: John Wiley \& Sons, Inc.; 2009:337-354.

62. Teijaro JR, et al. Endothelial cells are central orchestrators of cytokine amplification during influenza virus infection. Cell. 2011;146(6):980-991.

63. Camerer E, et al. Sphingosine-1-phosphate in the plasma compartment regulates basal and inflammation-induced vascular leak in mice. J Clin Invest. 2009;119(7):1871-1879

64. Shea BS, Brooks SF, Fontaine BA, Chun J, Luster $\mathrm{AD}$, Tager AM. Prolonged exposure to sphingosine 1-phosphate receptor-1 agonists exacerbates vascular leak, fibrosis, and mortality after lung injury. Am J Respir Cell Mol Biol. 2010;43(6):662-673.

65. Kaneider NC, et al. 'Role reversal' for the receptor PAR1 in sepsis-induced vascular damage. Nat Immunol. 2007;8(12):1303-1312.

66. Jones CA, et al. Slit2-Robo4 signalling promotes vascular stability by blocking Arf6 activity. Nat Cell Biol. 2009;11(11):1325-1331.

67. London NR, et al. Targeting Robo4-dependent Slit signaling to survive the cytokine storm in sepsis and influenza. Sci Transl Med. 2010;2(23):23ra19.

68. Okada Y, et al. A three-kilobase fragment of the human Robo4 promoter directs cell type-specific expression in endothelium. Circ Res. 2007; 100(12):1712-1722

69. Goolaerts A, et al. PAI-1 is an essential component of the pulmonary host response during Pseudomonas aeruginosa pneumonia in mice. Thorax. 2011;66(9):788-796.

70. Wiener-Kronish JP, Albertine KH, Matthay MA Differential responses of the endothelial and epithelial barriers of the lung in sheep to Escherichia coli endotoxin. J Clin Invest. 1991;88(3):864-875.

71. Ware LB, Matthay MA. Clinical practice. Acute pulmonary edema. NEnglJ Med. 2005;353(26):2788-2796.

72. Bastarache JA, Fremont RD, Kropski JA, Bossert FR, Ware LB. Procoagulant alveolar microparticles in the lungs of patients with acute respiratory distress syndrome. Am J Physiol Lung Cell Mol Physiol. 2009;297(6):L1035-L1041.

73. Fang X, Neyrinck AP, Matthay MA, Lee JW. Allogeneic human mesenchymal stem cells restore epithelial protein permeability in cultured human alveolar type II cells by secretion of angiopoietin-1. J Biol Chem. 2010;285(34):26211-26222.

74. Jiang D, et al. Regulation of lung injury and repair by Toll-like receptors and hyaluronan. Nat Med. 2005;11(11):1173-1179.

75. Folkesson HG, Matthay MA. Alveolar epithelial ion and fluid transport: recent progress. Am J Respir Cell Mol Biol. 2006;35(1):10-19.

76. Johnson MD, et al. Functional ion channels in pulmonary alveolar type I cells support a role for type I cells in lung ion transport. Proc Natl Acad Sci U S A. 2006;103(13):4964-4969.
77. Fang X, Fukuda N, Barbry P, Sartori C, Verkman AS, Matthay MA. Novel role for CFTR in fluid absorption from the distal airspaces of the lung. J Gen Physiol. 2002;119(2):199-207.

78. Planes C, Blot-Chabaud M, Matthay MA, Couette S, Uchida T, Clerici C. Hypoxia and beta 2-agonists regulate cell surface expression of the epithelial sodium channel in native alveolar epithelial cells. J Biol Chem. 2002;277(49):47318-47324.

79. Roux J, et al. Interleukin-1beta decreases expression of the epithelial sodium channel alpha-subunit in alveolar epithelial cells via a p38 MAPKdependent signaling pathway. J Biol Chem. 2005; 280(19):18579-18589.

80. Dada LA, Chandel NS, Ridge KM, Pedemonte C, Bertorello AM, Sznajder JI. Hypoxia-induced endocytosis of $\mathrm{Na}, \mathrm{K}$-ATPase in alveolar epithelial cells is mediated by mitochondrial reactive oxygen species and PKC-zeta. JClin Invest. 2003;111(7):1057-1064.

81. Iles KE, Song W, Miller DW, Dickinson DA, Matalon S. Reactive species and pulmonary edema. Expert Rev Respir Med. 2009;3(5):487-496.

82. Ware LB, Matthay MA. Alveolar fluid clearance is impaired in the majority of patients with acute lung injury and the acute respiratory distress syndrome. Am J Respir Crit Care Med. 2001;163(6):1376-1383.

83. Adamson IY, Bowden DH. The type 2 cell as progenitor of alveolar epithelial regeneration. A cytodynamic study in mice after exposure to oxygen. Lab Invest. 1974;30(1):35-42.

84. Kim CF, et al. Identification of bronchioalveolar stem cells in normal lung and lung cancer. Cell. 2005;121(6):823-835.

85. Rock JR, et al. Basal cells as stem cells of the mouse trachea and human airway epithelium. Proc Natl Acad Sci U S A. 2009;106(31):12771-12775.

86. McQualter JL, Yuen K, Williams B, Bertoncello I. Evidence of an epithelial stem/progenitor cell hierarchy in the adult mouse lung. Proc Natl Acad Sci US A. 2010;107(4):1414-1419.

87. Rawlins EL, et al. The role of Scgb1a1+ Clara cells in the long-term maintenance and repair of lung airway, but not alveolar, epithelium. Cell Stem Cell. 2009;4(6):525-534.

88. Chapman HA, et al. Integrin alpha6beta4 identifies an adult distal lung epithelial population with regenerative potential in mice. J Clin Invest. 2011;121(7):2855-2862

89. Kajstura J, et al. Evidence for human lung stem cells. N Engl J Med. 2011;364(19):1795-1806.

90. Uddin M, Levy BD. Resolvins: natural agonists for resolution of pulmonary inflammation. Prog Lipid Res. 2011;50(1):75-88.

91. Janssen WJ, et al. Fas determines differential fates of resident and recruited macrophages during resolution of acute lung injury. Am J Respir Crit Care Med. 2011;184(5):547-560.

92. Park D, et al. Continued clearance of apoptotic cells critically depends on the phagocyte Ucp 2 protein. Nature. 2011;477(7363):220-224.

93. Bratton DL, Henson PM. Neutrophil clearance: when the party is over, clean-up begins. Trends Immunol. 2011;32(8):350-357.

94. Narasaraju T, et al. Excessive neutrophils and neutrophil extracellular traps contribute to acute lung injury of influenza pneumonitis. Am J Pathol. 2011;179(1):199-210.

95. D'Alessio FR, et al. CD4+CD25+Foxp3+ Tregs resolve experimental lung injury in mice and are present in humans with acute lung injury. J Clin Invest. 2009;119(10):2898-2913.

96. Amato MB, et al. Effect of a protective-ventilation strategy on mortality in the acute respiratory distress syndrome. N Engl J Med. 1998;338(6):347-354.

97. [No authors listed]. Ventilation with lower tidal volumes as compared with traditional tidal volumes for acute lung injury and the acute respiratory distress syndrome. The Acute Respiratory Distress Syndrome 
Network. NEngl J Med. 2000;342(18):1301-1308.

98. Villar J, Kacmarek RM, Perez-Mendez L, AguirreJaime A. A high positive end-expiratory pressure, low tidal volume ventilatory strategy improves outcome in persistent acute respiratory distress syndrome: a randomized, controlled trial. Crit Care Med. 2006;34(5):1311-1318.

99. Webb HH, Tierney DF. Experimental pulmonary edema due to intermittent positive pressure ventilation with high inflation pressures. Protection by positive end-expiratory pressure. Am Rev Respir Dis. 1974;110(5):556-565

100.Dreyfuss D, Soler P, Basset G, Saumon G. High inflation pressure pulmonary edema. Respective effects of high airway pressure, high tidal volume, and positive end-expiratory pressure. Am Rev Respir Dis. 1988;137(5):1159-1164.

101. Parker JC, Townsley MI, Rippe B, Taylor AE, Thig pen J. Increased microvascular permeability in dog lungs due to high peak airway pressures. $J$ Appl Physiol. 1984;57(6):1809-1816.

102. Tremblay L, Valenza F, Ribeiro SP, Li J, Slutsky AS. Injurious ventilatory strategies increase cytokines and c-fos m-RNA expression in an isolated rat lung model. J Clin Invest. 1997;99(5):944-952.

103. Frank JA, Gutierrez JA, Jones KD, Allen L, Dobbs L, Matthay MA. Low tidal volume reduces epithelial and endothelial injury in acid-injured rat lungs. Am J Respir Crit Care Med. 2002;165(2):242-249.

104. Calfee CS, et al. Plasma receptor for advanced glycation end products and clinical outcomes in acute lung injury. Thorax. 2008;63(12):1083-1089.

105. Ranieri VM, et al. Effect of mechanical ventilation on inflammatory mediators in patients with acute respiratory distress syndrome: a randomized controlled trial. JAMA. 1999;282(1):54-61.

106. Parsons PE, et al. Lower tidal volume ventilation and plasma cytokine markers of inflammation in patients with acute lung injury. Crit Care Med. 2005;33(1):1-6

107. Imai Y, et al. Injurious mechanical ventilation and end-organ epithelial cell apoptosis and organ dysfunction in an experimental model of acute respiratory distress syndrome. JAMA. 2003;289(16):2104-2112.

108. Brower RG, et al. Higher versus lower positive end-expiratory pressures in patients with the acute respiratory distress syndrome. $N$ Engl J Med 2004;351(4):327-336

109. Meade MO, et al. Ventilation strategy using low tidal volumes, recruitment maneuvers, and high positive end-expiratory pressure for acute lung injury and acute respiratory distress syndrome: a randomized controlled trial. JAMA. 2008;299(6):637-645.

110. Mercat A, et al. Positive end-expiratory pressure setting in adults with acute lung injury and acute respiratory distress syndrome: a randomized controlled trial. JAMA. 2008;299(6):646-655.

111. Curley MA, et al. Effect of prone positioning on clinical outcomes in children with acute lung injury: a randomized controlled trial. JAMA. 2005; 294(2):229-237.

112. Taccone $\mathrm{P}$, et al. Prone positioning in patients with moderate and severe acute respiratory distress syndrome: a randomized controlled trial. JAMA 2009;302(18):1977-1984.

113.Papazian L, et al. Neuromuscular blockers in early acute respiratory distress syndrome. $N$ Engl J Med.
2010;363(12):1107-1116

114. Talmor D, et al. Mechanical ventilation guided by esophageal pressure in acute lung injury. $N$ Engl J Med. 2008;359(20):2095-2104.

115.Peek GJ, et al. Efficacy and economic assessment of conventional ventilatory support versus extracorporeal membrane oxygenation for severe adult respiratory failure (CESAR): a multicentre randomised controlled trial. Lancet. 2009 374(9698):1351-1363.

116. Derdak S, et al. High-frequency oscillatory ventilation for acute respiratory distress syndrome in adults: a randomized, controlled trial. Am J Respir Crit Care Med. 2002;166(6):801-808.

117. Diaz JV, Brower R, Calfee CS, Matthay MA. Therapeutic strategies for severe acute lung injury. Crit Care Med. 2010;38(8):1644-1650.

118. Staub NC. Pulmonary edema: physiologic approaches to management. Chest. 1978;74(5):559-564.

119. Prewitt RM, McCarthy J, Wood LD. Treatment of acute low pressure pulmonary edema in dogs: relative effects of hydrostatic and oncotic pressure, nitroprusside, and positive end-expiratory pressure. J Clin Invest. 1981;67(2):409-418.

120. Wiedemann HP, et al. Comparison of two fluidmanagement strategies in acute lung injury. NEngl JMed. 2006;354(24):2564-2575.

121. Wheeler AP, et al. Pulmonary-artery versus central venous catheter to guide treatment of acute lung injury. N Engl J Med. 2006;354(21):2213-2224.

122. Kuebler WM, Ying X, Singh B, Issekutz AC, Bhattacharya J. Pressure is proinflammatory in lung venular capillaries. J Clin Invest. 1999;104(4):495-502.

123. Calfee CS, et al. Plasma angiopoietin-2 in clinical acute lung injury: prognostic and pathogenetic

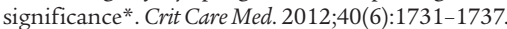

124. Gregory TJ, et al. Surfactant chemical composition and biophysical activity in acute respiratory distress syndrome. JClin Invest. 1991;88(6):1976-1981.

125. Spragg RG, et al. Effect of recombinant surfactant protein C-based surfactant on the acute respiratory distress syndrome. NEnglJMed. 2004;351(9):884-892.

126. Bernard GR, et al. High-dose corticosteroids in patients with the adult respiratory distress syndrome. N Engl J Med. 1987;317(25):1565-1570.

127. Meduri GU, et al. Effect of prolonged methylprednisolone therapy in unresolving acute respiratory distress syndrome: a randomized controlled trial. JAMA. 1998;280(2):159-165.

128. Steinberg KP, et al. Efficacy and safety of corticosteroids for persistent acute respiratory distress syndrome. NEngl J Med. 2006;354(16):1671-1684.

129. Meduri GU, et al. Methylprednisolone infusion in early severe ARDS: results of a randomized controlled trial. Chest. 2007;131(4):954-963.

130.Abraham E, et al. Liposomal prostaglandin E1 (TLC C-53) in acute respiratory distress syndrome: a controlled, randomized, double-blind, multicenter clinical trial. TLC C-53 ARDS Study Group. Crit Care Med. 1999;27(8):1478-1485.

131. Paine R 3rd, et al. A randomized trial of recombinant granulocyte-macrophage colony stimulating factor for patients with acute lung injury. Crit Care Med. 2012;40(1):90-97.

132. Bernard GR, et al. A trial of antioxidants $\mathrm{N}$-acetylcysteine and procysteine in ARDS. The Antioxidant in ARDS Study Group. Chest. 1997;112(1):164-172.

133. Ranieri VM, et al. Drotrecogin alfa (activat- ed) in adults with septic shock. $N$ Engl J Med. 2012;366(22):2055-2064.

134. Liu KD, et al. Randomized clinical trial of activated protein $\mathrm{C}$ for the treatment of acute lung injury. Am J Respir Crit Care Med. 2008;178(6):618-623

135. Taylor RW, et al. Low-dose inhaled nitric oxide in patients with acute lung injury: a randomized controlled trial. JAMA. 2004;291(13):1603-1609.

136. National Heart Lung, Blood Institute Acute Respiratory Distress Syndrome (ARDS) Clinical Trials Network, et al. Randomized, placebo-controlled clinical trial of an aerosolized beta-agonist for treatment of acute lung injury. Am J Respir Crit Care Med. 2011;184(5):561-568.

137. Smith FG, et al. Effect of intravenous beta-2 agonist treatment on clinical outcomes in acute respiratory distress syndrome (BALTI-2): a multicentre, randomised controlled trial. Lancet. 2012; 379(9812):229-235

138. Rice TW, et al. Enteral omega-3 fatty acid, gammalinolenic acid, and antioxidant supplementation in acute lung injury. JAMA. 2011;306(14):1574-1581.

139. Gupta N, Su X, Popov B, Lee JW, Serikov V, Matthay MA. Intrapulmonary delivery of bone marrow-derived mesenchymal stem cells improves survival and attenuates endotoxin-induced acute lung injury in mice. J Immunol. 2007;179(3):1855-1863.

140. Ortiz LA, et al. Interleukin 1 receptor antagonist mediates the antiinflammatory and antifibrotic effect of mesenchymal stem cells during lung injury. Proc Natl Acad Sci US A. 2007;104(26):11002-11007.

141.Lee JW, Fang X, Gupta N, Serikov V, Matthay MA. Allogeneic human mesenchymal stem cells for treatment of E. coli endotoxin-induced acute lung injury in the ex vivo perfused human lung. Proc Natl Acad Sci U S A. 2009;106(38):16357-16362.

142. Nemeth $\mathrm{K}$, et al. Bone marrow stromal cells attenuate sepsis via prostaglandin E(2)-dependent reprogramming of host macrophages to increase their interleukin-10 production. Nat Med. 2009;15(1):42-49.

143. Matthay MA, et al. Therapeutic potential of mesenchymal stem cells for severe acute lung injury. Chest. 2010;138(4):965-972.

144. Mei SH, et al. Mesenchymal stem cells reduce inflammation while enhancing bacterial clearance and improving survival in sepsis. Am J Respir Crit Care Med. 2010;182(8):1047-1057.

145. Islam MN, et al. Mitochondrial transfer from bonemarrow-derived stromal cells to pulmonary alveoli protects against acute lung injury. Nature Med. 2012;18(5):759-765.

146. Ott HC, et al. Regeneration and orthotopic transplantation of a bioartificial lung. Nat Med. 2010;16(8):927-933.

147. Ware LB, et al. Prognostic and pathogenetic value of combining clinical and biochemical indices in patients with acute lung injury. Chest. 2010;137(2):288-296.

148. Calfee CS, et al. Use of risk reclassification with multiple biomarkers improves mortality prediction in acute lung injury. Crit Care Med. 2011; 39(4):711-717.

149. Brodie D, Bacchetta M. Extracorporeal membrane oxygenation for ARDS in adults. $N$ Engl J Med. 2011;365(20):1905-1914.

150. Herridge MS, et al. Functional disability 5 years after acute respiratory distress syndrome. $N$ Engl J Med. 2011;364(14):1293-1304. 